\title{
Improving load balancing techniques by location awareness at indoor femtocell networks
}

\author{
A. Aguilar-Garcia ${ }^{1 *}$, S. Fortes ${ }^{1}$, A. Garrido², A. Fernandez-Duran² and R. Barco ${ }^{1}$
}

\begin{abstract}
Femtocells are the auspicious solution for the coverage and capacity challenges that mobile operators are currently facing indoors. Whereas some operators are focused on offloading data traffic from macrocells to femtocells, others prefer to ensure indoor coverage and prioritize voice traffic in order to mitigate the negative impact of blocked or dropped calls. In this context, this paper proposes a novel self-optimizing mechanism to prevent congestion problems at indoor environments, focused on operators that prioritize voice traffic connections. A challenge specific to femtocells is that they normally can only serve a small number of simultaneous connections. Based on this restriction, the proposed mechanism resizes femtocell coverage areas by tuning their transmission powers for load balancing purposes. How information on user location can enhance the optimization process is also investigated in this paper. According to this, two methods to estimate the received signal level per user are evaluated. Finally, the performance is studied by means of both simulations and a real field trial.
\end{abstract}

Keywords: Self-Organizing Networks (SON), Self-optimization, Mobility load balancing (MLB), Location awareness, Indoor, Femtocell

\section{Introduction}

The continuous advances in mobile technologies and personal devices (smartphones and tablets) have led to the deployment of heterogeneous networks (HetNets) to support the extreme traffic demand of new services. HetNets comprise different Radio Access Technologies (RAT) and several cell sizes (macrocell, microcells, picocell, etc.), resulting in a non-easily manageable complex mobile network infrastructure. In this sense, Self-Organizing Networks (SON) [1] have been identified by the 3rd Generation Partnership Project (3GPP) as a key feature to intelligently automate network management procedures in the future mobile networks. For that purpose, several self-x capabilities have been defined: self-configuration to automate network configuration and planning; selfoptimization to enhance network performance by automatically tuning its parameters; and self-healing to detect, identify, compensate, and recover failures in

\footnotetext{
* Correspondence: aag@ic.uma.es

1Universidad de Málaga, Andalucía Tech, Campus de Teatinos s/n, 29071

Málaga, Spain

Full list of author information is available at the end of the article
}

the network. In this context, several self-optimization mechanisms have been widely studied and analyzed in the literature and in European projects in both outdoor and indoor networks [2-8], with the aim of enhancing the network performance and increasing cell capacity and mobile network coverage.

SON techniques play an important role at indoor scenarios, since a high number of mobile connections are originated at home, work, shopping malls, etc. Those places normally present a poor signal quality that degrades the quality of service (QoS), the system coverage and capacity. To solve or reduce the impact of these indoor issues, operators are deploying plug and play, low-cost, short-range, and low-power cellular access points called femtocell access points (FAP) [9]. These devices are small versions of standard macrocells that work in the licensed frequency band and are connected to the operator core over the Internet (i.e., through enduser's broadband backhaul). The FAP coverage area is several meters, and the accessibility can be close (i.e., access is restricted to specific registered users) or open (i.e., access is allowed to any mobile subscriber, like in 
macrocells). As a consequence of its simple architecture and limited computational power, the maximum number of simultaneous connections is restricted to up to 64 users (even when radio resources are still available). Therefore, one of the most important shortcomings of femtocells is the connected-users limitations. Once the amount of active users reaches that top number, new connections (voice or data) that attempt to access the femtocell present a challenge from the operator perspective, as these connections could be blocked or accepted (in case another connection is handed over or dropped). That decision would depend on admission control (AC) scheme, which is not standardized. Note that this characteristic is independent of the scheduler, the availability of radio resources, or the circuit/packet-switched channel. Additionally, it is also applicable to any cellular technology (GSM, UMTS, or LTE).

On the one hand, the operator could be interested in a macrocell offload solution where macrocell data traffic hands over to femtocells (when possible) to increase network capacity. Hence, once the femtocell is full, the incoming voice calls that attempt to access are redirected to the macrocell, while the incoming data connections are accepted after handing over a voice traffic call to the macrocell. That situation could block many voice calls if the quality of the macrocell signal is poor. On the other hand, other operators could decide that femtocell deployments are aimed at enhancing the signal quality and extend the coverage at indoor environments where the macrocells present bad conditions (coverage holes, poor signal, etc.). According to this policy, the operator prefers that the voice calls are carried out through femtocells and data services hand over to the macrocell. The reason is related to the user point of view. Some operators consider that, once the femtocell is full, the client frustration is higher when a voice call is rejected than when he has no access to any other service. Therefore, the priority to accept an incoming voice call is higher than to accept data traffic. Consequently, those data connections hand over to the macrocell when an incoming voice call attempts to access a crowded femtocell. The impact of mobility failures on Voice over LTE (VoLTE) calls is discussed in the recent drive test presented in [10], where the handover failure rate (HFR) for pedestrian users is over $21 \%$, which is an unacceptable user experience for operators.

Additionally, nowadays, there is a growing interest both in academia and in industry in indoor positioning techniques, due to the expansion of smart devices. In this field, the final aim is to achieve a seamless solution for location-based services (LBS). Although several technologies provide indoor localization, there is a compromise between the system accuracy and the cost. The better the accuracy of the localization system, the more expensive the system is. That indoor accuracy varies from millimeters (e.g., ultra-wide band (UWB)) to several meters (e.g., radio-frequency identification (RFID)).

Location information could extremely enhance the mobile network performance as it could support network management mechanisms in real-time. However, current operation, administration, and management (OAM) architectures usually manage network parameters in periods of hours or even days which could be inefficient in dynamic scenarios such as indoor environments. In this sense, the hybrid architecture presented in [11] is one possible solution for implementing real-time selfmanagement mechanisms.

This paper is focused on developing SON algorithms for indoor scenarios where operators prioritize voice connections over any other service. In addition, the algorithms are supported by indoor localization information to enhance the network performance in real-time. According to this, the main contribution of this work is the deployment of a novel location-based load balancing method that prevents or immediately reduces temporal overloaded situations at indoor femtocell environments when operator prioritizes voice traffic. The proposed system dynamically modifies femtocell transmission power based on the number of connected users, users' location, and RSS values to resize the network cell coverage. These RSS values are obtained from two different sources: current measurement reports (MR) or historical RSS measurements. Thus, the two methods were performed. A detailed study was carried out in a realistic heterogeneous simulated scenario and a field trial in order to evaluate both systems.

The rest of this paper is organized as follows. Section 2 presents an overview of the state of the art associated to this work. Section 3 formulates the mobility load balancing use case at indoor scenarios. Section 4 describes the design and the scheme of the proposed method. Section 5 discusses and compares the simulations results while the evaluation in a real deployment is presented in Section 6. Section 7 summarizes the main conclusions.

\section{Related work}

The self-optimization use case of mobility load balancing (MLB) has been proposed by 3GPP [12]. MLB use case aims to share users from overloaded cells to their low loaded neighboring cells in order to gain network capacity and improve overall QoS. Several techniques for MLB have been proposed by both academia and industry [12-16]. Those techniques tune network parameters to reach a better configuration that alleviates the congestion situation outdoors. Focusing on indoor environments, the work proposed in [17] presented a new planning strategy for placing femtocells and making the most of automatic traffic sharing algorithms for a 
LTE heterogeneous network. Femtocells are plug and play devices connected to the operator's network by a broadband connection (e.g., cable or xDSL). Thus, these networks are prone to unplanned deployment in many cases (the client is free to locate the femtocell anywhere). The authors in [18], proposed a method to adjust hysteresis margins depending on an estimation of the distance from the base station to the user equipment (UE), reduces the number of redundant handovers (HOs) while keeping the throughput of femtocells as high as possible. The work shown in [19] studied the persistent congestion problems on traffic distribution in LTE femtocell enterprise scenario. That work implemented and compared several traffic sharing algorithms that tune femtocell transmission power and handover margins, following a fuzzy logic controller (FLC) scheme in order to automatically adjust femtocell parameters. Nevertheless, temporarily overloaded cells issues were not addressed in that work. The study of [20] analyzed the importance of the femtocell capacity in terms of the number of active users for mobility load balancing in temporal overloaded situations. However, the user location was not considered in that study.

None of the previous studies made use of the user location for MLB in indoor scenarios. The authors in [21] introduced location information into the load balancing mechanisms to reduce handovers or call blocking rates in temporarily overloaded cells by modifying the coverage area. However, it was focused on outdoor UMTS macrocell networks. Other studies utilized users' positioning to enhance self-optimization mechanism and reduce costs [22-25], but those works are not in the scope of MLB use case.

The techniques proposed in this paper are based on resizing cell areas by modifying transmission power. In this context, reference [23] introduced the received signal strength (RSS) fingerprint as a method for cellular optimization and compared the RSS fingerprint with a propagation model to predict the received signal power. However, this work was oriented to outdoor environments. Another study [26] was focused on femtocell networks and presented a balancing data traffic method based on users' location and RSS information by resizing cell area, but it did not analyze some special characteristics of femtocells (e.g., the number of active users) which is more restricted than the occupied radio resources. In addition, it was in line with the first operators' policy in Section 1, i.e., using femtocells mainly for data traffic.

\section{Problem description}

Femtocells are proposed as a solution to solve some of the current cellular challenges: the UE battery lifetime is increased and the user Quality of Experience (QoE) is enhanced due to the proximity between femtocells and users, while operators reduce capital and operational expenditure (CAPEX/OPEX). Conversely, femtocells present some shortcomings that must be addressed, for example, the unpredictable occasional events that could cause unexpected overload conditions in the network. These temporal variations, combined with the coverage holes and time-variant fading caused by reflections and obstacles, could negatively affect network performance.

Generally, voice and data traffic, as well as local user densities, vary in temporal and spatial domain. Those situations lead to degraded indoor cellular networks where many people could want to use their mobile devices at the same time, close to the same area and/or for a short period. For example, at the airport, people waiting for a delayed flight at the boarding gate could collapse a femtocell or, a celebrity walking through a mall where everybody is interested in taking pictures and sharing them instantaneously in social networks or calling friends to share the experience. To support these extreme situations, a simple solution could be to plan the network resources according to the peak traffic. However, this solution would increase operator costs.

MLB algorithms, by adaptive cell sizing, are the most suitable solution to avoid these cell congestions. Such algorithms are based on optimizing different parameter settings in order to adjust service areas (e.g., cell transmission power). Consequently, connections are handed over from the problematic serving cell to the most suitable neighboring cell, sharing traffic and balancing the load of the network. The estimation of the best configuration parameters can be formulated as a classical optimization problem [21]. However, operators prefer solving these issues by heuristic rules as the full information required to build analytical models are rarely available.

Temporal network congestions must be solved from two perspectives. On the one hand, from the user point of view, the performance is measured according to their QoE, which can be improved by minimizing two network indicators: the global call blocking ratio (CBR) and the call dropping ratio (CDR). CBR is defined as ratio of the number of calls that attempt to access the network but fail $\left(N_{\text {blocked_calls }}(\Delta t)\right)$ to the number of calls that attempt to access the network ( $N_{\text {attempted_- }}$ calls $(\Delta t))$ in a period $\Delta t$ :

$$
\begin{aligned}
& \operatorname{CBR}(\Delta t)=\frac{N_{\text {blocked }_{\text {calls }}}(\Delta t)}{N_{\text {attempted }_{\text {calls }}(\Delta t)}} \\
& =\frac{N_{\text {blocked }_{\text {calls }}}(\Delta t)}{N_{\text {blocked }_{\text {calls }}}(\Delta t)+N_{\text {accepted }_{\text {calls }}}(\Delta t)}
\end{aligned}
$$

where $N_{\text {accepted_calls }}(\Delta t)$ is the number of accepted calls in the period $\Delta t$.

While the CDR is defined as the ratio of the number of active calls that are not finished by the user but due 
to a network issue like a handover failure, bad coverage, congestion, etc. $\left(N_{\text {dropped_calls }}(\Delta t)\right)$ to the number of calls that are accepted in the network in a period $\Delta t$ :

$$
\operatorname{CDR}(\Delta t)=\frac{N_{\operatorname{dropped}_{\text {calls }}}(\Delta t)}{N_{\text {accepted }_{\text {calls }}}(\Delta t)}
$$

To summarize this information into one indicator that measures the users' dissatisfaction, the user dissatisfaction ratio (UDR) is used. It is interpreted as a combination of CBR and CDR [19], which is also an important parameter from the operator point of view because the users' QoE is important to keep clients.

$$
\operatorname{UDR}(\Delta t)=\operatorname{CBR}(\Delta t)+(1-\operatorname{CBR}(\Delta t)) \cdot \operatorname{CDR}(\Delta t)
$$

On the other hand, from the operator point of view, in addition to the previous indicators, an important criterion is also given by the amount of signaling data necessary to control the network fluctuations. A key indicator to measure that information is user handover ratio (UHR) described as the number of handovers $N_{\text {hando- }}$ vers $(\Delta t)$ over the accepted calls in the time span $\Delta t$. Low values of UHR are desirable and it can be defined as:

$$
\operatorname{UHR}(\Delta t)=\frac{N_{\text {handovers }}(\Delta t)}{N_{\text {accepted }_{\text {calls }}}(\Delta t)}
$$

In this work, the proposed method aims to optimize the user QoE by finding a good trade-off between CBR/ CDR and UHR.

\section{Load balancing algorithm}

\subsection{Information sources}

The aim of the proposed algorithm is to resize the cell coverage areas in order to share traffic between neighboring cells. For that reason, the main network parameter that will be tuned is the cell transmission power. To address this operation, the received power per mobile phone is used by the system in order to calculate the new cell transmission power. That information could be obtained or estimated from different sources (propagation models, measurement reports, etc.). Radio propagation models, as empirical mathematical formulas, characterize radio wave propagations. These models are very sensitive to pedestrian, obstacles, etc., which vary both in time and space, above all at indoor environments. Other sources such as measurement reports provide instantaneous information about RSS, while historical power measurements provide an estimation of RSS based on the previous recent RSS measurements. These RSS values could be different depending on the source due to the influence of fading and shadowing that could dramatically alter the RSS along time. In consequence, this work is focused on the analysis and comparison of an average estimation of the RSS versus instantaneous values in order to determine their benefits. Therefore, instantaneous RSS information from measurement reports and from historical RSS information (estimated based on "historical path loss information") per position are studied. Note that the second approach requires the users' location to create the historical path loss database, whereas location is not used by the first approach.

Regarding the users' location, a recent advanced research provides accurate indoor-positioning techniques which could enhance SON mechanisms. In this sense, the mobile devices could be well located (location error below $1 \mathrm{~m}$ ), thanks to external network localization processes like infrared laser and UWB. The drawback is that these kinds of systems are very expensive due to their low location error and high accuracy. Systems such as RFID and Wi-Fi could reduce these expenses although the position accuracy is degraded (location error of meters).

In order to manage all this information, to reduce traffic congestions, and to implement the mechanisms, the local-centralized architecture described in [11] is the base of the proposed system.

A brief description about RSS from the measurement reports, as well as the methodology and mechanisms to get the RSS based on historical RSS information and the users' location, are described in the next subsections.

\subsubsection{Instantaneous RSS from measurement reports}

Mobile devices provide valuable information about the network conditions through the measurement reports (MR). These reports are periodically sent to the cell and contain information about the channel quality (current mobile transmitted/received power, block error ratio of the data channel, etc.). This is vital to assist dynamic network planning and radio resource management (RRM) processes in power control decisions and handover. 3GPP specifications define this type of measurements in $[27,28]$.

The received signal power (i.e., RSS) per mobile phone is included in the measurement reports, although named in a different way depending on the radio technology. For $2 \mathrm{G}$ deployments, it is called received signal level (RxLev) whereas, for 3G, it is named as received signal code power (RSCP) and in LTE as reference signal received power (RSRP). That information is periodically forwarded to the OAM layer from the base stations. In practice, the mobile network does not support continuous real-time communication to the OAM elements due to the amount of signaling data and the high-level location of the OAM in the hierarchy. For that reason, this real-time process is difficult to be implemented nowadays. However, recent OAM architectures [11] place some of its functions in lowest levels, e.g., sites, making 
this real-time process viable. This is in line with distributed SON architectures proposed by 3GPP for future networks [1].

\subsubsection{Historical path loss maps (HPLM) mechanism}

The RSS information depends on several factors such as propagation channel, cell transmission power, and user location. However, since the signal path loss (PL) is independent of the cell transmission power, the path loss information will be used instead of the RSS information to create a database. Path loss values are calculated and stored into this database together with the measured localization to build the so named HPLM. The relationship between both parameters is given by

$$
\operatorname{RSS}_{\text {cell }}(x, y)=\operatorname{PTX}_{\text {cell }}-\mathrm{PL}_{\text {cell }}(x, y)
$$

where $\operatorname{RSS}_{\text {cell }}(x, y)$ is the received power at $(x, y)$ position from cell, $\mathrm{PTX}_{\mathrm{cell}}$ is the equivalent isotropically radiated power (EIRP) of cell and $\mathrm{PL}_{\text {cell }}(x, y)$ is the radio signal path loss at point $(x, y)$ from cell. In this context, in order to support SON mechanisms, the database contains the path loss per position received from each base station. This information is calculated based on the MRs (i.e., RSS values) performed by the UEs over time, the network configuration parameters (i.e., cells transmission power) and the users' positions. Thanks to this HPLM database, the system will be able to get an estimation of the RSS per position based on Eq. (5).

Building the HPLM database is a continuous process composed by two different kinds of information (Fig. 1a). On the one hand, the measurements reported from the active users are used to calculate the current path loss information from each cell $\mathrm{PL}_{\text {cell }}(i, j)_{u}$, where coordinates $(i, j)_{u}$ are the position of user $u$. On the other hand, and synchronized with the measurements reports, the active user position $(i, j)_{u}$ is supplied to the mechanisms by an external indoor-positioning system [29] (the implementation of an indoor-positioning system is out of the scope of this work). With these data (measurement and location), the average path loss for a position and cell is updated according to the following equation:

$$
\widehat{\mathrm{PL}}_{\text {cell }}(i, j)=\frac{1}{N} \sum_{m=1}^{N} \mathrm{PL}_{\text {cell }}(i, j)_{m}
$$

where $\mathrm{PL}_{\text {cell }}(i, j)_{m}$ are the different path loss measurements over time at position $(i, j)$ and $N$ is the total number of measurements. That $\widehat{\mathrm{PL}}_{\text {cell }}(i, j)$ would be used to estimate the $\widehat{\operatorname{RSS}}_{\text {cell }}(i, j)$ according to Eq. (5).

The radio channel conditions, above all at indoor environments, suffer continuous changes due to the number of people, obstacles, etc. Therefore, the number of samples, $N$, to build the estimated path loss value per position should be a configurable parameter and could be quite different depending on the scenario and the position. For this work, in order to keep the propagation channel conditions updated, the number of samples is limited in time:

$$
N(x, y)=N_{\text {samples }}^{T_{\text {interpolation }}}(x, y)
$$

where $N_{\text {samples }}^{T}(x, y)$ is the number of samples at coordinates $(x, y)$ within a period $T_{\text {interpolation }}$.

Note that, at the beginning of the process, no historical information is stored in the database. Hence, this mechanism is under a state of standby where no information about the path loss is provided to any selfoptimization algorithm (the network is not optimized). Additionally, even when a wide range of data is stored, there could be positions with no path loss information as $\left(x_{p}, y_{p}\right)$ in Fig. 2 . In this case, the mechanism needs to guess a possible path loss value of these positions. To address this problem, that information is calculated through an interpolation.

According to this, the path loss value per cell $\widehat{\mathrm{PL}}$ $\left(x_{p}, y_{p}\right)_{\text {cell }}$ is estimated as

$$
\widehat{\mathrm{PL}}_{\text {cell }}\left(x_{p}, y_{p}\right) \approx \sum_{m=1}^{R} w_{m} \cdot \widehat{\mathrm{PL}}_{\text {cell }}\left(x_{m}, y_{m}\right)
$$

where $R$ is the number of positions with stored path loss information, $\widehat{\mathrm{PL}}_{\text {cell }}\left(x_{m}, y_{m}\right)$ is the estimated path loss

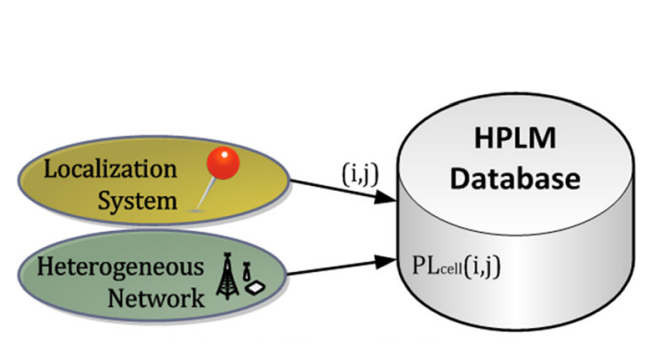

a) Acquisition of information

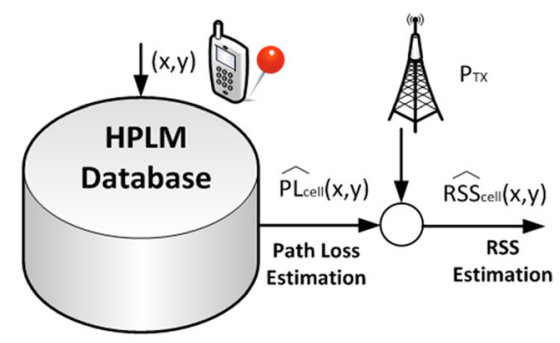

b) Estimation of RSS value

Fig. 1 HPLM scheme. a Acquisition of information. $\mathbf{b}$ Estimation of RSS value. 


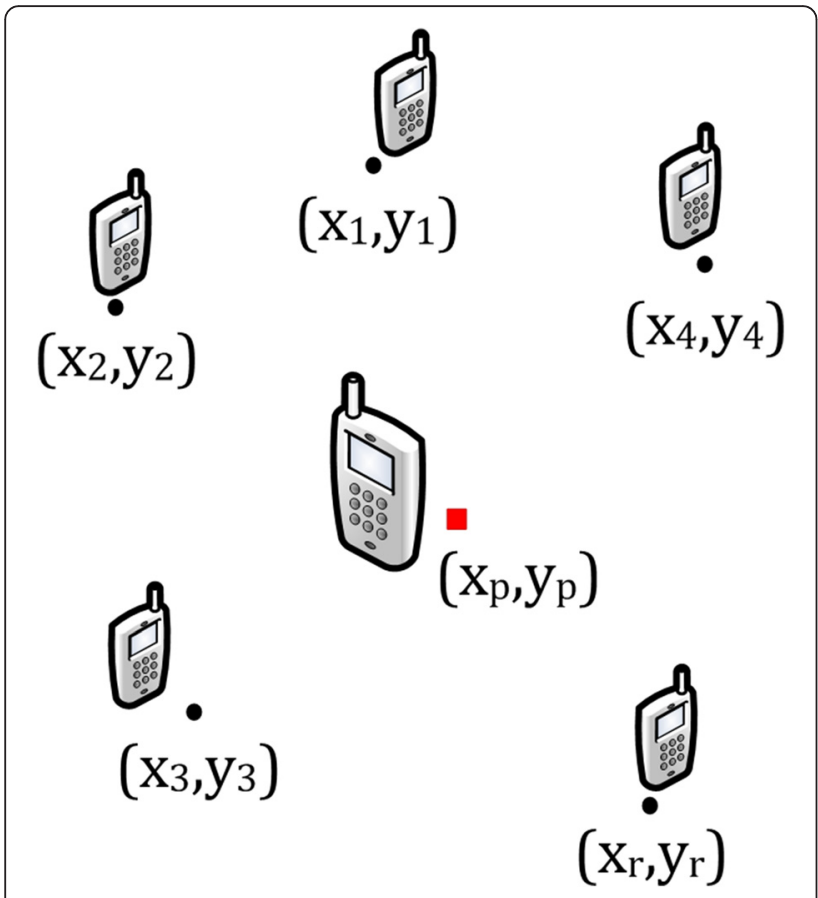

Fig. 2 Interpolation at coordinates $\left(x_{p}, y_{p}\right)$

value of the position $m$ that is already stored in the database and $w_{m}$ is the weight assigned to each $m$ position depending on its distance to the studied UE. These weights must be inversely proportional to the distance between the known locations and the UE position in order to give more importance to those path loss values closer to the UE position. The weights are computed as follows:

$$
w_{m}=\frac{\frac{1}{d_{m-p}}}{\sum_{i=1}^{R} \frac{1}{d_{i-p}}}
$$

where $d_{a-b}$ is the distance from position $a$ to position $b$ and $R$ is the number of the stored path loss values. The range of $m$ would be from 1 to $R$ and the sum of these $w_{m}$ weights is 1 .

\subsection{Load balancing mechanism}

RSS information is obtained from the HPLM database depending on the user location (Fig. 1b). Alternatively, when location is not available or positioning system statistical parameters are unsuitable for the algorithm (high location error), RSS information is directly obtained from the UE measurement reports. Subsequently, RSS information is used as input of an iterative rule-based mechanism to offload the congested cells by adjusting the femtocell-transmitted powers.

This mechanism is proposed to provide a consistent solution to those operators aiming at prioritizing voice traffic users over data traffic users in indoor femtocell environments, as the impact to the end user is more frustrating in voice calls than in data traffic. For that purpose, the system ensures free resources for voice traffic when the indoor network is congested, whereas data traffic is handed over to macrocells or suffers outage for a while (that would be managed by the $\mathrm{AC}$ or the schedulers). In other words, the mechanism reduces the call blocking ratio to the minimum value as well as the call dropping ratio when network parameters are modified in order to balance and enhance network performance.

The proposed load balancing algorithm is shown in Fig. 3. First, the system examines the percentage of users per femtocell $L_{\text {cell }}$ based on its users' capacity:

$$
L_{\text {cell }}=\frac{N_{\text {connected }} \text { cell }}{N_{\text {femtocels }}}
$$

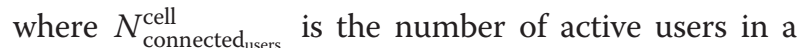
femtocell cell and $N_{\text {femtocell_capacity }}$ is the maximum number of simultaneous connections that this femtocell supports.

All $L_{\text {cell }}$ are ranked in decreasing order to create vector $\vec{L}$ :

$$
\vec{L}=\left.\left(L_{1}, L_{2} \ldots L_{i} \ldots L_{N_{\text {fentocells }}}\right)\right|_{L_{i} \geq L_{i+1}}
$$

The next step consists of selecting the first item of that vector $L_{s=1}$, i.e., the most congested femtocell, to start the balance process. Hereafter that selected femtocell will be called studied cell $(s)$. That value $L_{s}$ is compared with a fixed threshold $\alpha$; therefore, the algorithm only starts when the ratio of connected users is over $\alpha$. In that case, that femtocell should be offloaded. This $\alpha$ value is defined by the operator according to their policies and priorities.

Now, the neighboring femtocells of the studied cell $s$ are selected from the neighbor cell lists. Since, normally, there is a high overlapping between femtocells because of their unplanned deployments, these neighbor cell lists usually include a lot of femtocells. Then, the users' load per neighboring femtocell of the studied cell $s$ is evaluated. The idea is to determine whether these neighboring cells have space to allocate new users or not. In this sense, the average neighbor femtocell load is calculated as:

$$
A_{s}=\frac{\sum_{a=\text { neighboring feemtocell }} L_{\text {cell }_{a}}}{N_{\text {neighboring }}}
$$

where $L_{\text {cell } \_a}$ was previously calculated according to Eq. (10), cell_ $a$ represents the cell identification of the neighboring cells and $N_{\text {neighboring_femtocells }}$ is the number of neighboring femtocells. 


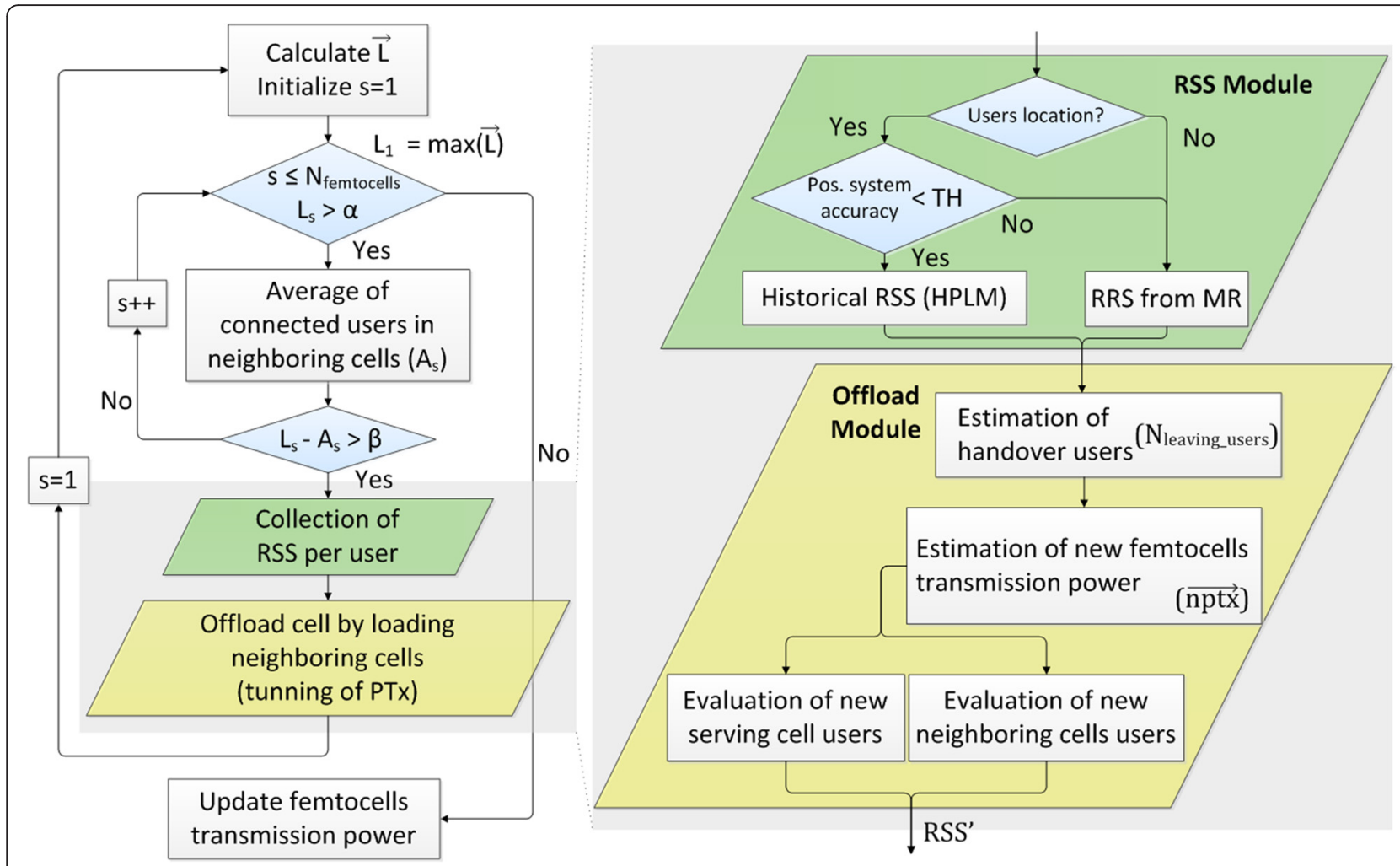

Fig. 3 Power user balancing algorithm scheme

After that, the situation could be:

- $A_{s} \geq L_{s}$, which means that the studied cell has less users than the average users of its neighboring cells. Therefore, no users should leave that cell because the situation out of this cell is the same or worst.

- $A_{s}<L_{s}$, which means that the situation of the serving cell could be critical but it could be solved as, in average, its neighbors present lower level of users' load. In consequence, some users of the serving cell will hand over to the most suitable neighboring cells.

To accomplish good QoE and avoid ping-pong effects, a threshold $\beta$ limits the minimum difference between those previous indicators $\left(A_{s}, L_{s}\right)$. This means that the difference between the ratio of connected users from the serving cell $\left(L_{s}\right)$ and the average ratio of selected serving cell neighbors $\left(A_{s}\right)$ should be over $\beta$ to evaluate that overloaded situation and to continue with the algorithm. Otherwise, a new cell $(s=s$ +1 ) is selected to be analyzed (new studied cell), and the algorithm goes back to the previous steps, as Fig. 3 shows. This process would stop when $s$ is over the total number of femtocells.

Once the studied cell $s$ has been selected to be offloaded, it is necessary to determine the RSS value per user in that cell (by means of the RSS module-green box in Fig. 3) prior to estimate the number of users that should handover to neighboring cells and to modify the transmission power of the cells (by means of the offload module-yellow box in Fig. 3).

\subsubsection{RSS module (green module)}

As Section 4.1 has introduced, two ways to obtain the RSS information are analyzed and evaluated as part of this algorithm:

- RSS from HPLM: this method is the preferred information source. However, users' location is required to estimate the value of its RSS. In this case, Eq. (5) estimates those values of RSS for each UE position and femtocell, based on the stored HPLM and Eqs. (6) and (8).

- RSS from MR: the RSS information is directly acquired from the mobile reports. It provides actual data about the propagation channel.

The selection of one method or the other would depend on the availability of the users' location. However, the positioning system could introduce a location error defined by statistical parameters (mean error, standard deviation, etc.). This shortcoming will degrade the network performance; therefore, the positioning system accuracy is 
compared to a threshold, $\mathrm{TH}$ (parameter), to decide whether to continue with the HPLM or to change to MR method.

Finally, this RSS module provides a vector $\overrightarrow{\mathrm{RSS}_{\text {user }}}$ for each UE with its received power from both the serving cell and the neighboring cells:

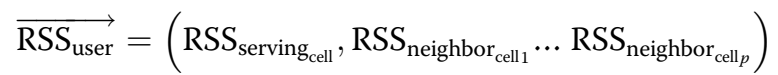

When there is no information about the received power from a given neighboring cell, the RSS for such cell is set to $-130 \mathrm{dBm}$.

\subsubsection{Offload module (yellow module)}

Once the RSS information is received from the RSS module, the next step is to estimate the number of users that should leave the studied serving cell $\left(N_{\text {leavingusers }_{\text {us }}}^{\text {cell }}\right)$ and to update femtocell transmission power to complete the handovers of these users to the more suitable neighbor cell. In this context, the algorithm tries to balance the number of users in the serving cell based on the average number of connected users ( $\left.N_{\text {users_average }}\right)$ in the neighboring cells according to:

$$
N_{\text {leaving }_{\text {users }}}^{\text {cell }}=\text { ceil }\left(N_{\text {connected }_{\text {users }}}^{\text {cell }}-N_{\text {users }_{\text {average }}}\right)
$$

where ceil function gets the nearest integer towards infinity, $N_{\text {connected }}^{\text {cersers }}$ is the number of connected users at the studied serving cell and $N_{\text {users_average }}$ is defined as:

$$
N_{\text {users }_{\text {average }}}=\frac{\sum_{a=\text { cell }_{\text {id }}} N_{\text {connected users }^{a}}^{a}}{1+N_{\text {neighboring }_{\text {femtocells }}}}
$$

where $a$ identifies the cell_id of the serving cell and each neighboring femtocell and $N_{\text {neighboring_femtocells }}$ is the number of these neighboring femtocells.

The next step is to determine the transmission power adjustment that must be applied in the femtocells to accomplish the desired network behavior. For that purpose, the algorithm in Fig. 4 is executed. The procedure Offload(RSS, $N_{\text {leaving_users }}$ ) calculates the new femtocell transmission power, $\overrightarrow{\mathrm{nptx}}$, according to the number of users that should hand over to other femtocells. In this sense, for each user $m$, the mechanism determines the new transmission power of the neighboring femtocell to handover that user. It is based on the strongest value of RSS from neighboring cells of all the connected users in the studied cell. Until the condition in line 17 is not accomplished, the next strongest value of RSS from neighboring cells of all the connected users in the studied cell is deleted from the RSS matrix in order to select the next strongest one in the following iteration. In general, the selected neighboring cell $c$ and the user $u$ would be different in each loop.

The script is repeated until that requirement is fulfilled. Then, the offset is calculated, i.e., the parameter involved in the offset to accomplish a handover (offset_handover) (e.g., event A3 in LTE [27]) and the vector $\overrightarrow{\mathrm{nptx}}$ is updated with the new transmission power of cell $c$. Finally, the new transmission power of the studied cell (serving cell) is evaluated based on previous

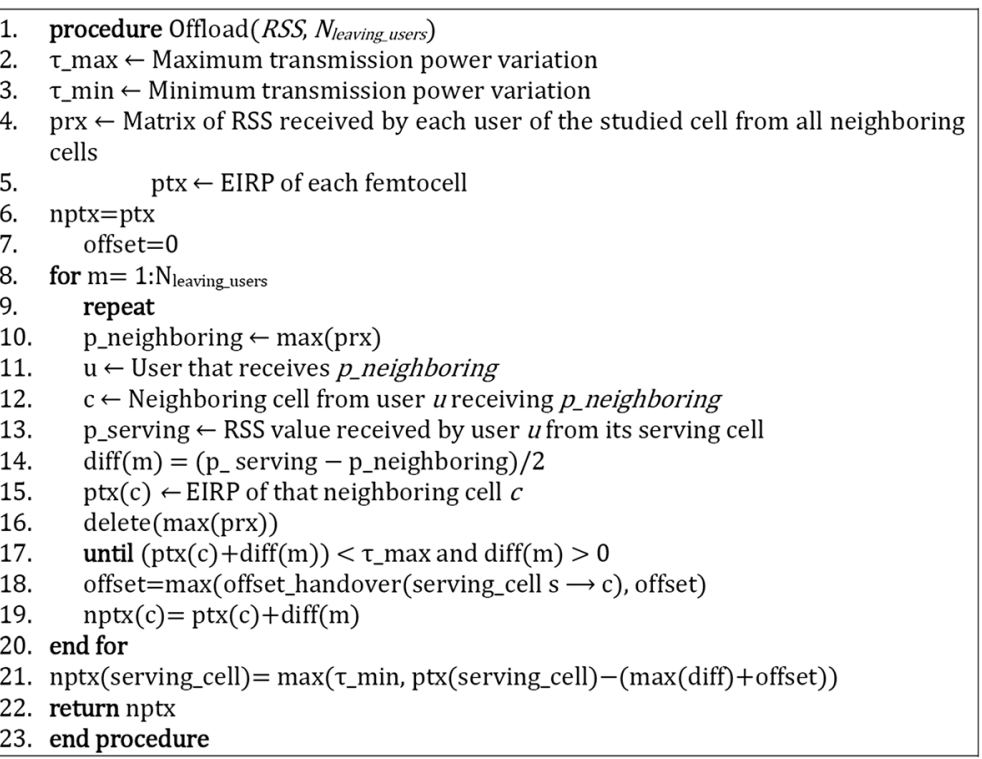

Fig. 4 Offload process 
transmission power variations $\overrightarrow{\mathrm{diff}}$ and the maximum value of offset. The procedure returns the vector $\overrightarrow{n p t x}$. Note that the decrease of the serving cell transmission power is truncated by $\tau_{\min }$. This condition is required to guarantee the QoS and, thus, to avoid dropped calls and to successfully terminate the voice calls.

After that, and based on vector $\overrightarrow{\mathrm{nptx}}$, the system evaluates the new network configuration to estimate how the network could be when femtocells are tuned. In order to do that, new values of RSS would be calculated (RSS') as well as the ratio of connected users to each femtocell $(L$ ' cell $)$. To determine those values of RSS', the PTX cell of Eq. (5) is replaced by nptx(cell), i.e., the new EIRP of that femtocell. In the same way, the new percentage of users per femtocell $L^{\prime}$ cell (see Eq. (10)) is calculated. The new number of active users per $\operatorname{cell}_{i}\left(N_{\text {connected }}^{\prime}\right.$ sers cell $)$ is estimated based on the number of users that accomplish: $\left\{\operatorname{RSS}_{\text {cell }_{i}}^{\prime}>\operatorname{RSS}_{\text {cell }_{j}}^{\prime} \forall i \neq j\right\}$.

To conclude, the flow goes back $(s=1)$ to the beginning of the algorithm (see Fig. 3) to analyze the new network situation (before the new parameters are set on the femtocells) and determine whether the network is wellbalanced or another femtocell needs to be analyzed because there are still overload issues.

Next sections evaluate the proposed methods. Furthermore, an analysis of the algorithm based on each RSS model is carried out. These methods are evaluated with a system-level simulator, as well as in a real field-test.

\section{Simulation analysis}

\subsection{Analysis set-up}

Firstly, the implementation of the proposed mechanism and its assessment is accomplished in a dynamic LTE system-level simulator [30]. For this paper, a single trisectorized macrocell (blue triangle) has been deployed into a large scenario of $3 \mathrm{~km}$ long and $2.6 \mathrm{~km}$ wide where Málaga Airport has been designed and located $500 \mathrm{~m}$ far from the macrocell as a realistic simulated indoor environment. The building is 265 long and $180 \mathrm{~m}$ wide. To avoid border effects, the wrap-around technique has been implemented. This description is illustrated in the red dotted line of the left picture of Fig. 5. Inside the airport, 12 femtocells have been deployed (red dots) to ensure coverage signal from, at least, two femtocells at all points of the scenario. In this context, interference introduced by the macrocell inside the airport is also taken into account, as the airport is close enough to the macrocell.

Additionally, the simulator implements a propagation model that considers several environment configurations: Winner II project [31]. It models the propagation conditions for indoor, outdoor, outdoor-to-indoor, and indoor-to-outdoor scenarios. Likewise, shadowing is modeled by a spatially correlated log-normal distribution with different standard deviation for outdoor and indoor users, whereas fast-fading is modeled by the Extended Indoor A (EIA) model for indoor users [32].

The movement of mobile users is based on the approach presented in [33], a random waypoint mobility model. Each walking path has been assigned with a different probability in order to create hotspots along the simulation where network congestions may occur.

The simulator also includes common RRM features, such as cell reselection (CR), HO based on "A3" and "A5" events, directed retry (DR), and scheduler. Further details about the simulator configuration are summarized in Table 1, while a full description is detailed in [30].

As the proposed method is focused on temporary congestion situations and prioritize voice traffic, the algorithm must evaluate the network indicators in short periods. Additionally, the period to change the transmission power in a femtocell (i.e., to set the configuration file) usually takes tens of seconds. Consequently, the epoch to launch

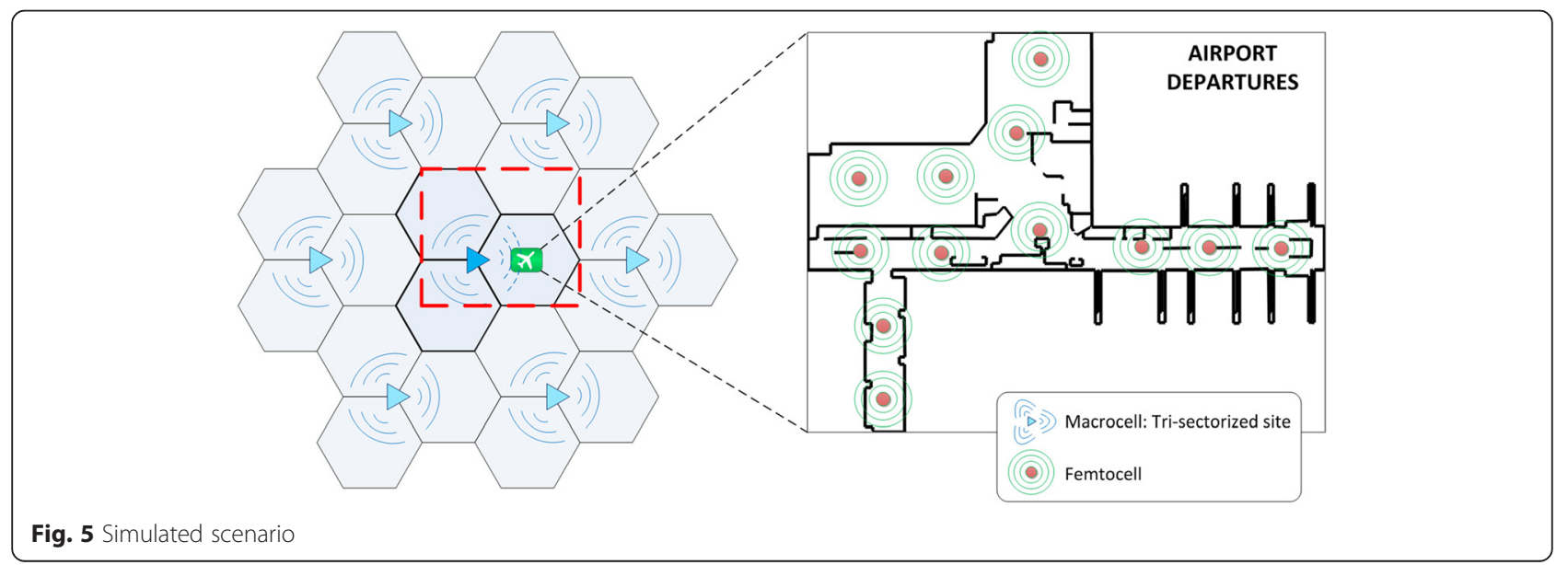


Table 1 Simulation parameters [30]

\begin{tabular}{|c|c|c|}
\hline \multirow[t]{4}{*}{ Load balancing algorithm } & Epoch time & $1 \mathrm{~min}$ \\
\hline & $a$ & $50 \%$ \\
\hline & $\beta$ & $20 \%$ \\
\hline & $\mathrm{T}_{\text {interpolation }}$ & $60 \mathrm{~min}$ \\
\hline \multirow[t]{4}{*}{ Propagation model } & Outdoor-outdoor & Winner II C2 \\
\hline & Outdoor-indoor & Winner II C4 \\
\hline & Indoor-indoor & Winner II A1 \\
\hline & Indoor-outdoor & Winner II A2 \\
\hline \multirow[t]{3}{*}{ Base station model } & EIRP & 3 (femto)/43 (macro) dBm \\
\hline & Directivity & Omni (femto)/tri-sector (macro) \\
\hline & Access & Open (femto)/open (macro) \\
\hline \multirow[t]{2}{*}{ Mobile station model } & Noise figure & $9 \mathrm{~dB}$ \\
\hline & Noise density & $-174 \mathrm{dBm} / \mathrm{Hz}$ \\
\hline \multirow[t]{2}{*}{ Traffic model } & Calls & Poisson (avg. 0.43calls/user · h) \\
\hline & Duration & Exponential (avg. 100 s) \\
\hline \multirow[t]{2}{*}{ Mobility model } & Outdoor & $3 \mathrm{~km} / \mathrm{h}$, random direction \& wrap-around \\
\hline & Indoor & Random Waypoint \\
\hline Service model & Voice over IP & $16 \mathrm{kbps}$ \\
\hline \multirow[t]{5}{*}{ RRM model } & Bandwidth & $5 \mathrm{MHz}$ (25 PRBs) \\
\hline & Access control & Directed retry (threshold $=-44 \mathrm{dBm}$ ) \\
\hline & Cell reselection & Criteria S, R \\
\hline & Handover & Events $\mathrm{A} 3, \mathrm{~A} 5$ \\
\hline & Scheduler & $\begin{array}{l}\text { Time domain: round-robin } \\
\text { Frequency domain: best channel }\end{array}$ \\
\hline Time resolution & & $100 \mathrm{~ms}$ \\
\hline
\end{tabular}

the proposed mechanism is set to $60 \mathrm{~s}$ while the simulation lasts $24 \mathrm{~h}$.

Moreover, in order to simplify the AC and the scheduler, once a femtocell allocates its maximum number of active users, any new call attempt is rejected (although thanks to the directed retry, it could be allocated into another femtocell) and only voice traffic (VoLTE) is considered in the simulations.

Examples of HPLM are drawn in Fig. 6 for some of the deployed cells. Initially, beacons were placed each $2 \mathrm{~m}$ to emulate the transient response and to gather RSS samples at those positions. In addition, the resolution of the HPLM is set to $50 \mathrm{~cm}$. Thus, those locations with no path loss information at the beginning are estimated based on Eq. (8) of the interpolation procedure previously explained. At the beginning of the tests, as Table 1 shows, macrocells have been set up to transmit $43 \mathrm{dBm}$ and femtocells $3 \mathrm{dBm}$. Over the simulation time, femtocells change those values in order to optimize the network. Note that these adaptations do not modify previous HPLM because the path loss is independent of the system parameters (e.g., transmit power). To ensure that all locations are always simultaneously covered by at least two stations, the minimum value of transmission power variation is limited to $-30 \mathrm{~dB}$. The maximum increment is set to $10 \mathrm{~dB}$ as the femtocell maximum transmission power is restricted to $13 \mathrm{dBm}$, and they are initially configured at $3 \mathrm{dBm}$.

\subsection{Performance assessment methodology}

The proposed MLB algorithm is compared with the non-optimized network (baseline) and the power traffic sharing (PTS) algorithm presented in reference [19] which tunes femtocell transmission power based on comparing the call blocking ratio of a cell to the average call blocking ratio of its neighboring cells. To quantify the advantages and disadvantages of the MLB algorithm, the indicators described in Section 3 are assessed: UDR and UHR.

In addition, a sensitivity study of $\alpha$ and $\beta$ has been performed to obtain the lowest value of UDR for this scenario, showing homogeneous results (around $2 \%$ ) for low values of these parameters. The best values are $\alpha=$ 50 and $\beta=20$. 


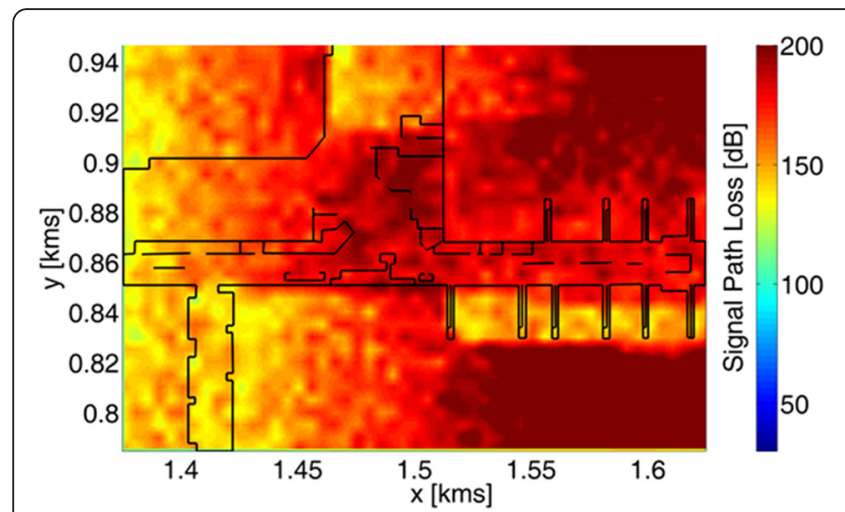

a) Macrocell| I (Sector 1)

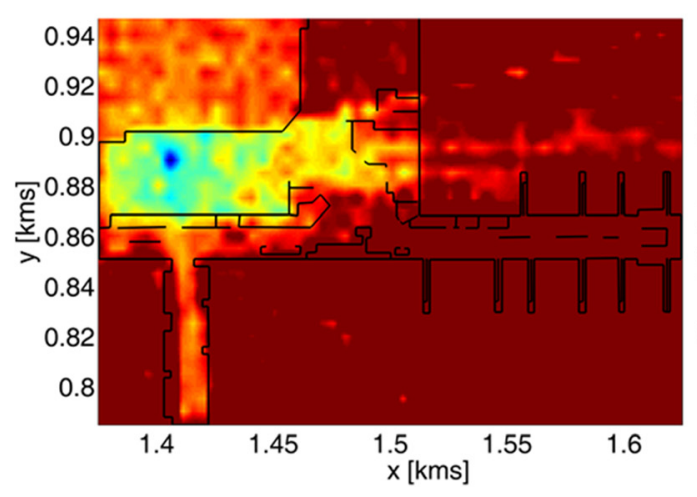

c) Femtocell IV

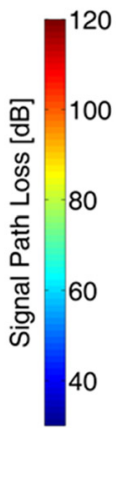

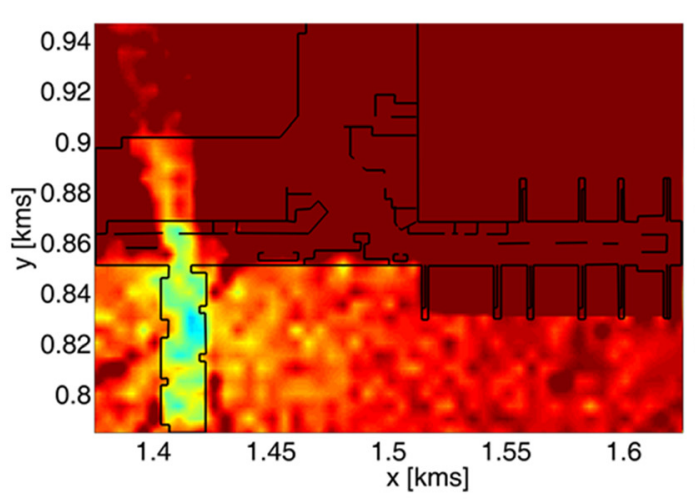

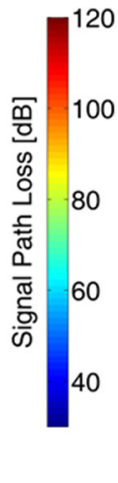

b) Femtocell II
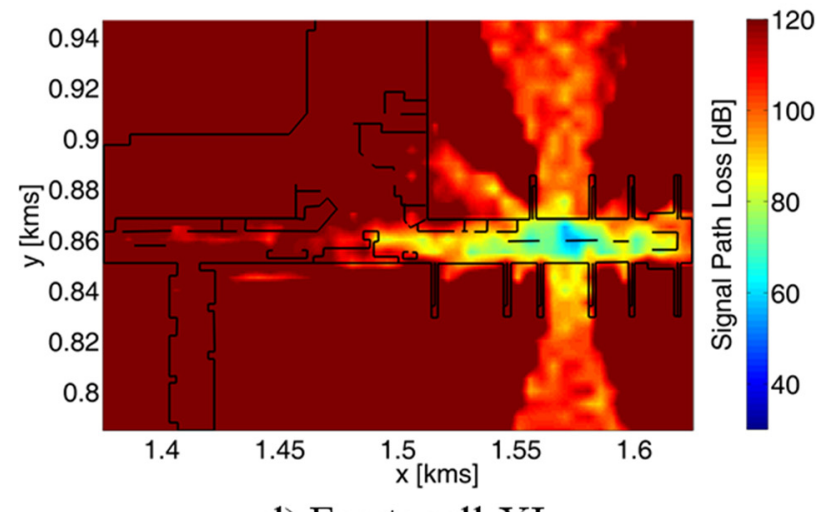

d) Femtocell XI

Fig. 6 Example of HPLM (dB). a Macrocell (sector I). b Femtocell II. c Femtocell IV. d Femtocell XI

\subsection{Assessment of the results}

The simulation results are described in this subsection. In order to accomplish an extensive and complete study, the evaluation of the algorithms was performed for four different processing capacities (i.e., maximum number of connected users allowed by the femtocell: 4, 8, 16, and 32 users). The number of users in the scenario has been established according to the analyzed cell capacity, e.g., for a femtocell capacity limit of 4 users, a number of 500 users per hour is simulated, whereas for a femtocell limit of 8 , a population of 1000 users per hour is defined.

\subsubsection{Limitation of 4 users per femtocell}

In this case, the capacity of femtocells is restricted to a maximum of four connected users at the same time. As Fig. 7 on the left shows, the non-optimized network (baseline) presents an average UDR of around $11 \%$. This value is not acceptable for any mobile operator since it is a critical situation that must be fixed as soon as possible. The PTS algorithm reduces the average UDR, as expected. However, with PTS, the temporary network congestions are not rapidly optimized because of its long convergence period (further details in [19]). Conversely, the yellow bar represents the proposed mechanism

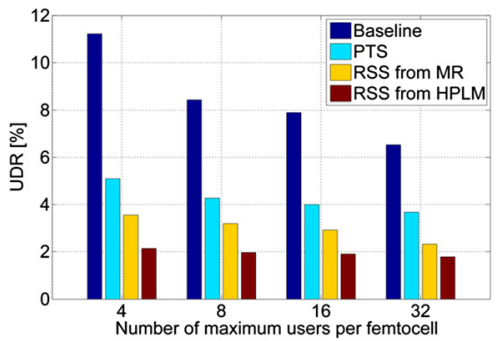

a) Users Dissatisfied Ratio [\%]

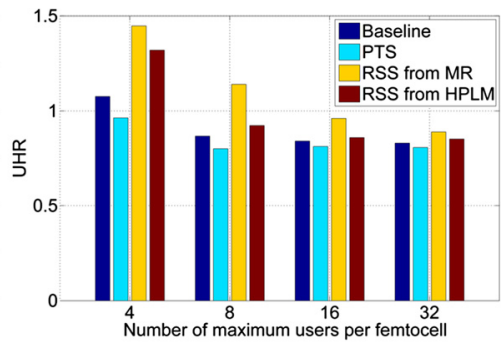

b) Number of HO per user

Fig. 7 Network performance (24 h). a Users dissatisfied ration (\%). b Number of HO per user 
supported by the MRs. This method reduces the average UDR to around $3.5 \%$ by reducing the percentage of blocking calls. Moreover, the HPLM method presents even better users' satisfaction. The relative improvement is, in average, around $40 \%$ compared with the MR method. The reason of this enhancement is related with multi-path reflections and wall obstacles that present severe fast variations of the propagation channel (fast - fading $\Delta f \approx[ \pm 5 \mathrm{~dB}])$. This means, the estimated transmission power adaptation in the MR method could be affected by the fast-fading when the number of users is low, as a consequence of the channel instability.

On the other hand, Fig. $7 \mathrm{~b}$ presents the UHR indicator. As expected, resizing femtocell coverage area involves an increase in network signaling and the number of handovers. Nevertheless, that increment in the number of handovers is negligible compared with the users' satisfaction enhancement.

\subsubsection{Limitation of 8 users per femtocell}

In this scenario, the femtocell capacity has been doubled (up to eight active users) as well as the number of pedestrian sitting/walking through the airport corridors. Fig. 7a illustrates similar behavior as the previous scenario. In this case, the baseline presents an average UDR around $8.5 \%$ and the PTS method reaches an average UDR value of $4 \%$. Meanwhile, the proposed algorithm reduces this value to $3 \%$ (MR) and to $2 \%$ (HPLM). Note that the average performance of both methods (MR and HPLM) is closer than in the previous scenario (4 users).

From the point of view of the number of handovers, baseline, PTS, and HPLM method is quite similar. However, the MR method presents more number of handovers in average.

\subsubsection{Limitation of 16 users per femtocell}

As in the previous scenario, both the femtocell capacity and the number of UEs in the airport have been doubled. For this scenario, as the number of active users is higher, the average UDR value for both methods (HPLM and MR) is closer (see Fig. 7a). The same behavior is observed from the handovers point of view (see Fig. 7b).

\subsubsection{Limitation of $\mathbf{3 2}$ users per femtocell}

Finally, the femtocells with the highest users processing capacity are evaluated. In this case, the number of handovers to be managed is increased. Therefore, the impact of the fading in the MR method is reduced as the new transmission power adaptations would trigger the handover process for most selected users. This means the ratio of successful handovers is higher when the femtocell capacity is increasing. However, for the HPLM method, once the database has a wide variety of RSS samples, the performance would not be improved as much as in the case of the MR method when increasing the number of users. According to this, Fig. 7a shows how the average UDR values of both methods are closer, less than $2 \%$ for HPLM and over $2 \%$ for MR. Baseline keeps high UDR around $7 \%$. UHR depicts comparable number of handovers, therefore, similar signaling load.

To summarize, it has been shown how problematic temporary network congestion could be managed to enhance users' satisfaction. The proposed algorithm provides valuable enhancement in the operator network over the non-optimized situation and outperforms the PTS algorithm. The HPLM method is stable in the four scenarios, whereas the MR method improves when the femtocell capacity is higher and the number of UEs is increased. This means, the higher the number of UEs is, the more similar the average RSS from MR is, compared to RSS from HPLM method.

In any case, the HPLM method outperforms the MR method when the influence of the location accuracy has not been addressed. Next subsection assesses the performance of the HPLM method when the positioning system provides the user location with some degree of inaccuracy.

\subsection{Error in user positioning}

The positioning system could have some inaccuracy in the user localization. Consequently, the RSS information obtained from the HPLM might be wrong. This situation is evaluated in this study to assess the robustness and reliability of the proposed HPLM method in indoor femtocell environments.

To emulate this situation, the users' location error is modeled as a normal distribution $\left(\mu, a^{2}\right)$ where $\mu=\{25,50,100,200,400,800\} \mathrm{cm}$ and $\sigma={ }^{\mu} / 3 \mathrm{~cm}$, being the mean and the standard deviation, respectively. According to this, Fig. 8 depicts the same four previous scenarios where the users' location accuracy is modified in relation to the precision error supplied by different positioning systems. The average value of the UDR over 24-h simulation for the HPLM method is illustrated for each average location error (continuous blue line) and scenario. The average value of the UDR for the MR method (dotted green line) is also presented in the figures.

For all the scenarios, as expected, the users' satisfaction decreases when the average location error increases. The top left figure (max. 4 users/femtocell) shows the good performance of the HPLM method compared to the MR method when the average location error is less than $5 \mathrm{~m}$. In other case (average location error over $5 \mathrm{~m}$ ), the MLB algorithm should get the RSS information from the MR method. Next figure, top right (max. 8 users/femtocell), presents similar behavior. Now, the algorithm should avoid HPLM method when the average location error is over $4 \mathrm{~m}$. 


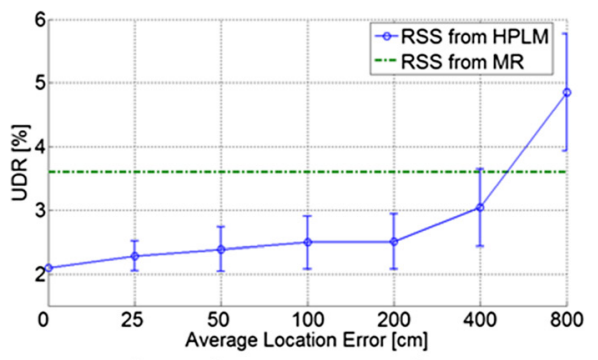

a) Maximum connected users: 4

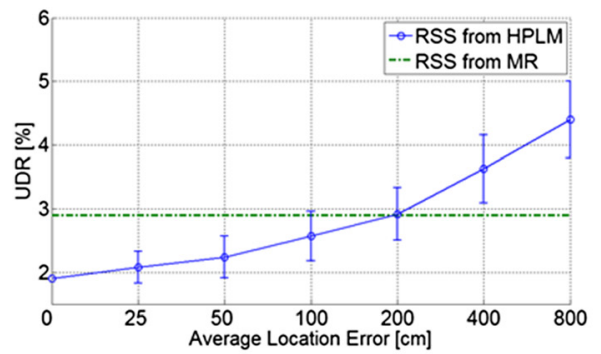

c) Maximum connected users: 16

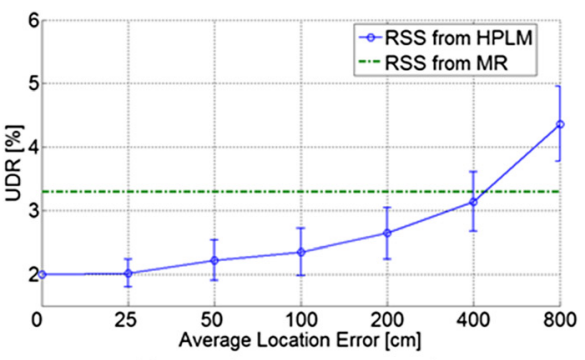

b) Maximum connected users: 8

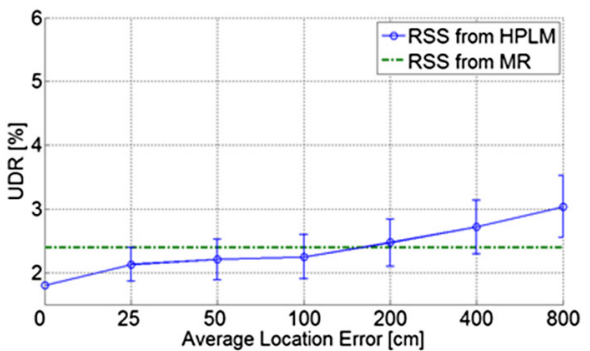

d) Maximum connected users: 32

Fig. 8 UDR based on different location errors. a Maximum connected users: 4. b Maximum connected users: 8. c Maximum connected users: 16. d Maximum connected users: 32

The bottom left figure continues with similar pattern but both methods reach the same UDR value sooner. Therefore, in this case, the MR method should be followed when the average location error is over $2 \mathrm{~m}$. The same trend can be observed in the bottom right figure, where the HPLM method outperforms the MR method for average location error under $1.5 \mathrm{~m}$.

The results are performed with information from 100 simulations for each location error and use case. The $95 \%$ confidence interval is around $\pm 0.25 \%$ of UDR for any use case with $25 \mathrm{~cm}$ of location error. It is extended when location error is increased: from around $\pm 1 \%$ for maximum 4 users/femtocell to $\pm 0.5 \%$ for maximum 32 users/femtocell.

These positioning accuracies are not very demanding and could be achieved with several indoorlocalization systems like the one described in [34] where the 90th percentile error of the proposed method is lower than $3 \mathrm{~m}$ and the average location error is $1.5 \mathrm{~m}$. Other smartphone-based indoorlocalization systems, like those cited in [29], improved the average location error to $1 \mathrm{~m}$, thanks to the integration of the sensor information into their localization engine [35].

To summarize, this study has demonstrated that the increase in the location error degrades the algorithm performance when it uses the HPLM method to get the RSS information. The HPLM outperforms the MR method for low average location errors; however, that threshold would depend on the type of femtocell.

\section{Test-bed analysis}

\subsection{Field-test description}

The next step was the evaluation of the proposed algorithm in a real network. The 3G Alcatel Lucent

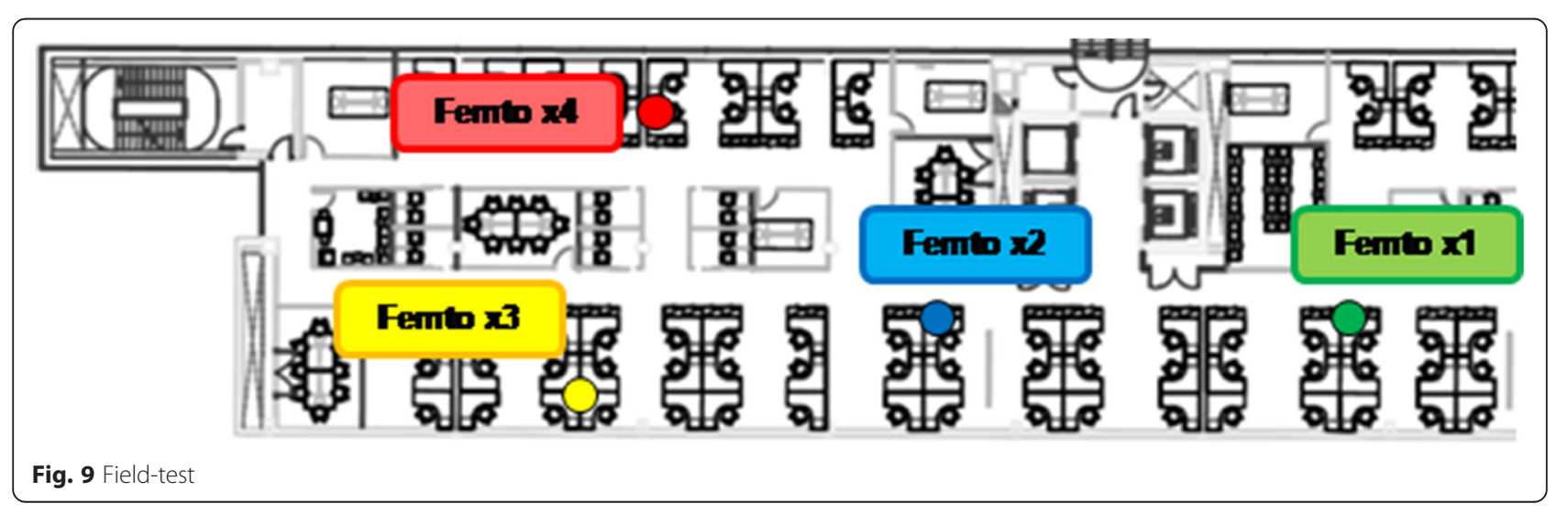




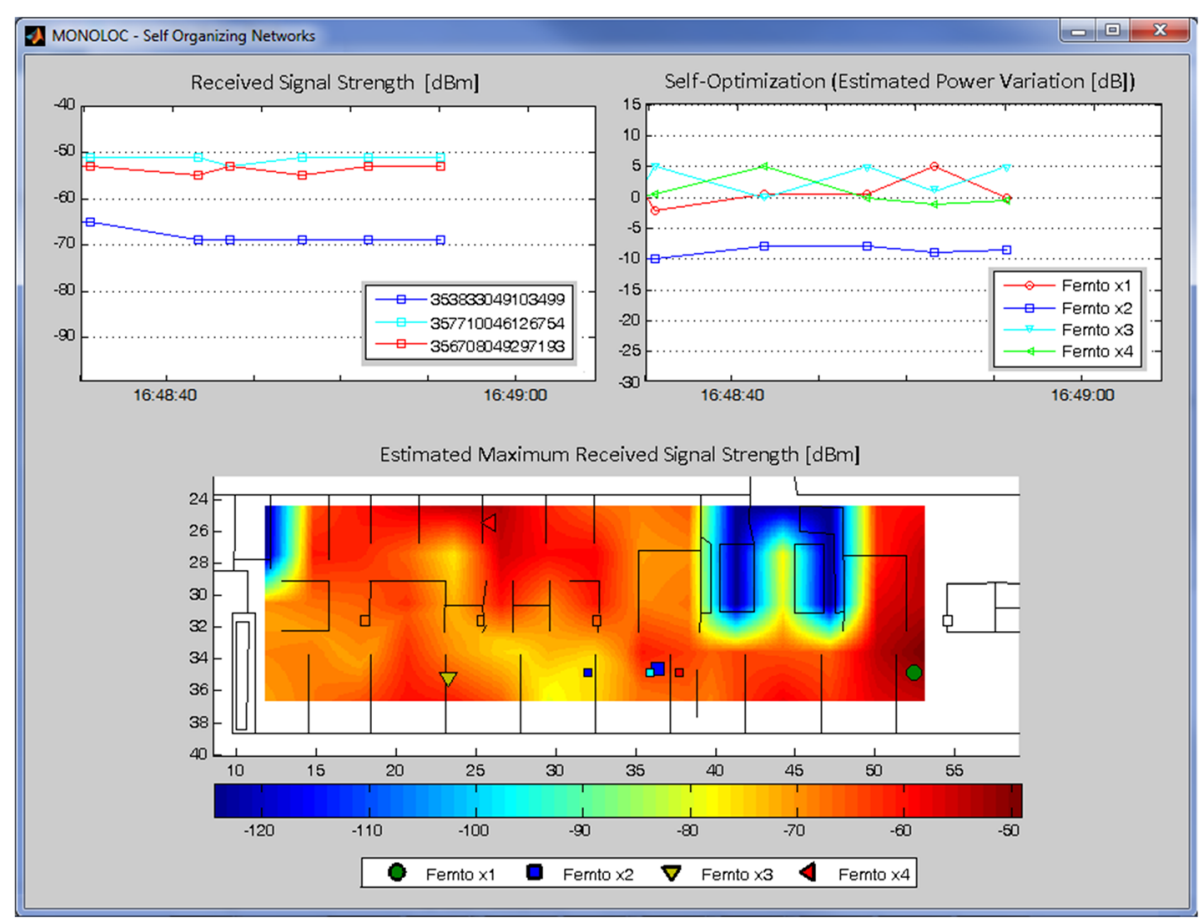

Fig. 10 Graphical user interface (GUI)

9361 Home Cell v2 model [36] has been selected as femtocell. Four of them were deployed into an office building and connected to a core network to verify the proposed algorithm and compare both methods to obtain the RSS values. The femtocell distribution along the building is depicted in Fig. 9. All femtocell transmission power was initially set to $3 \mathrm{dBm}$ and, subsequently, they were tuned according to the algorithm outputs. Here, femtocell capacities are restricted to maximum four connected users at the same time.

To evaluate the proposed algorithm, three mobile phones were allocated in the same cell (Femto x2) to verify how the femtocell coverage areas are modified to share these users to/from a neighboring femtocell/the serving cell.

\subsection{Field-test evaluation}

Due to the complexity and the constraints to set the network parameters on the mobile operator infrastructure, a simple use case was proposed to evaluate the algorithm.

For monitoring what is happening in the femtocell network, a simple graphical user interface (GUI) has been developed as Fig. 10 illustrates. It is composed of three parts:

- Received signal strength $[\mathrm{dBm}]$ : This window (top left) illustrates the RSS value from the serving cell (the icon identifies the femtocell) for each mobile phone (the color identifies the user) in real-time.

- Self-optimization (estimated power variation $[\mathrm{dB}]$ ): It depicts the self-optimizing algorithm response
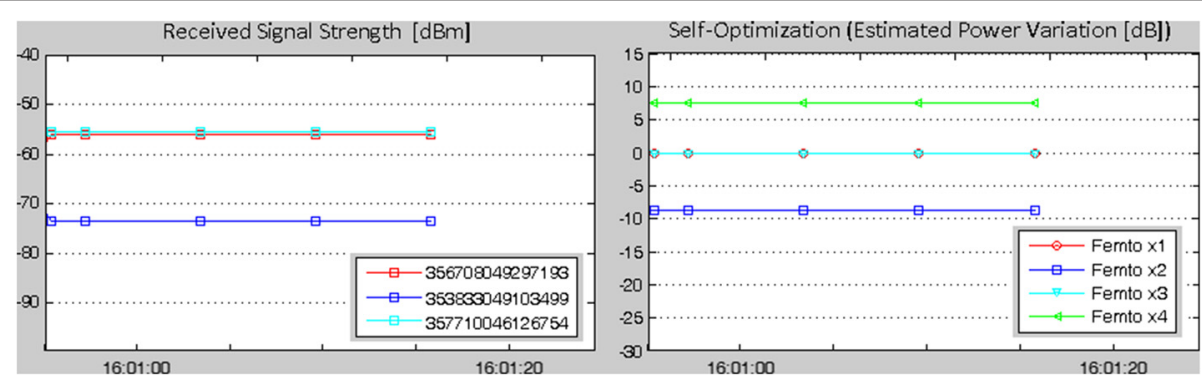

Fig. $11 \mathrm{HPLM}$ method 


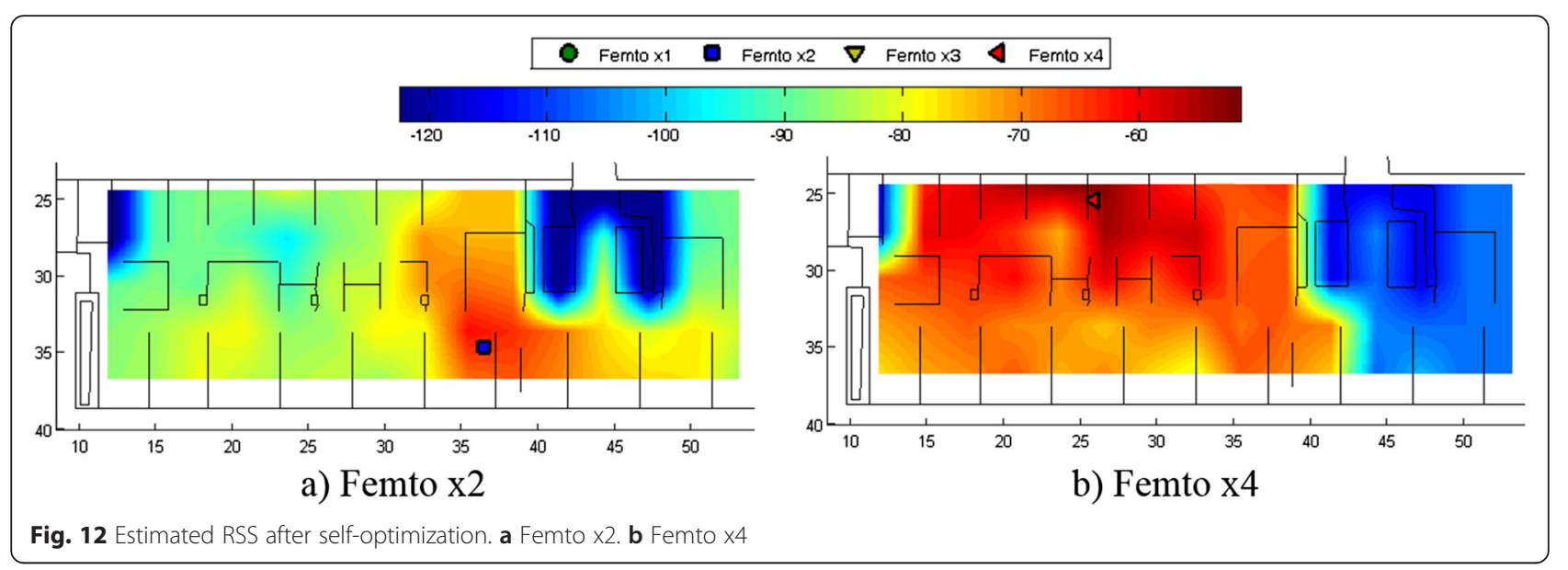

(top right). In this case, it is the transmit power variation that should be set up per femtocell to balance the network. Each femtocell is identified by an icon and a color (see bottom bar of Fig. 10).

- Estimated maximum RSS [dBm]: The scenario is showed in this window (bottom) where femtocells and mobile phones positions are also integrated. The highest estimated values of RSS per position are represented on the scenario.

The three mobile phones were located on a fixed position, as the bottom image of the Fig. 10 shows (three small squares as they are connected to Femto x2). As previously explained, the color identifies the mobile phone and the shape of the icon indicates its serving cell, in this case, Femto $\mathrm{x} 2$ (square) for all of them. The user location is manually introduced in the system to avoid location errors. The algorithm analyzes the network situation every $5 \mathrm{~s}$ (minimum period to ensure QoS in the field test). The MLB algorithm based on both methods (HPLM and MR) is checked under these conditions.

On the one hand, the algorithm supported by the HPLM method is analyzed in Fig. 11. For this situation, the algorithm is triggered because a femtocell (Femto $\mathrm{x} 2$ ) is overloaded due to the fact that three active users are attached to it, occupying the $75 \%$ of the femtocell capacity $L_{\text {femto } x 2}>$ $50 \%\left(\alpha=50 \%, \beta=20 \%\right.$ and $T_{\text {interpolation }}=60 \mathrm{~min}$, $)$ while the neighboring cells are empty $A_{\text {femto } x 2}=0 \%$. The estimated value of the power adaptation of each femtocell is showed in the top right of Fig. 11. Notice that, as the left window illustrates, the RSS values (per user) provided by this method are quite similar in time. The reason is related to the short time in which the RSS values are estimated (every $5 \mathrm{~s}$ ) in comparison to the number of samples (over 500) that are taken to calculate these estimated RSS values (see Eq. (5)); therefore, small changes in RSS values are insignificant between close RSS estimations.

The new configuration is set on the femtocells through a configuration file to update femtocell transmission power. After that, the femtocell network is monitored again to validate the modifications.

As expected, the blue (left icon in Fig. 10) mobile phone hands over to Femto x4, whereas the other two stay on the same serving cell (Femto $\mathrm{x} 2$ ). After this, the self-optimization window does not depict any data because the network is balanced and the algorithm is not triggered (until an overloaded situation appears again). The new estimated RSS values of Femto $\mathrm{x} 2$ and Femto $\mathrm{x} 4$ are illustrated in Fig. 12.

On the other hand, the MR method is evaluated in Fig. 13 where the same problematic situation is deployed.

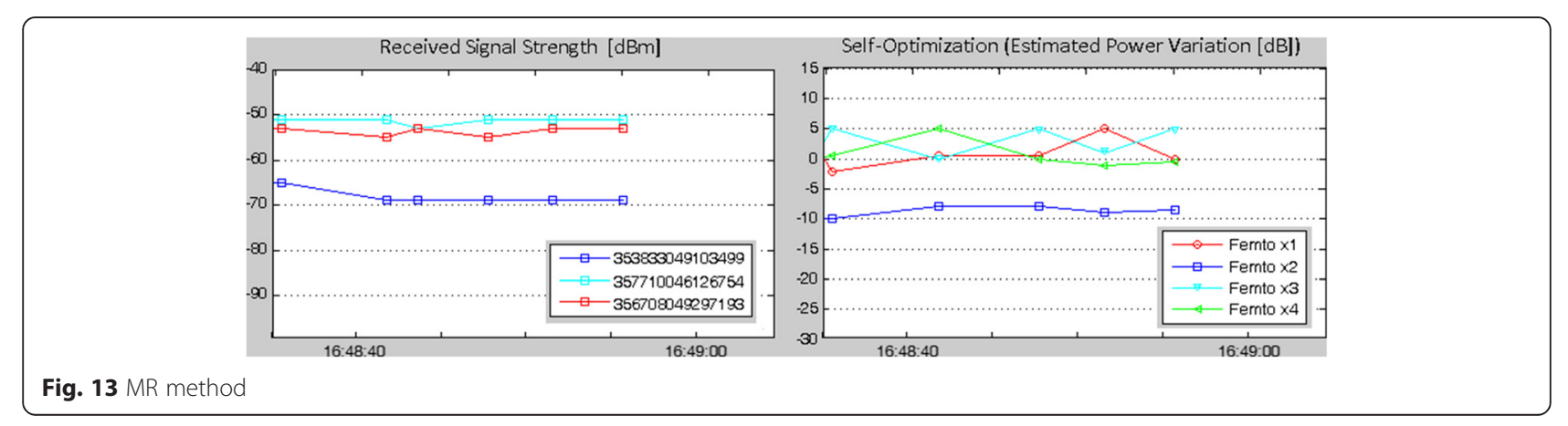


Table 2 HPLM performance

\begin{tabular}{llllll}
\hline & \multicolumn{2}{l}{$\begin{array}{l}\text { HPLM method } \\
\text { (no location error) }\end{array}$} & & \multicolumn{2}{l}{$\begin{array}{l}\text { HPLM method } \\
\text { (location error = 3 m) }\end{array}$} \\
\cline { 2 - 3 } & $\Delta$ Power & Users & & APower & Users \\
\hline Femto_x1 & 0 & 0 & & 0 & 0 \\
Femto_x2 & -9.0 & 2 & & -8.8 & 2 \\
Femto_x3 & 0 & 0 & & 0 & 0 \\
Femto_x4 & 7.5 & 1 & & 7.3 & 1 \\
\hline
\end{tabular}

In this case, as previously described, the RSS values fluctuate over time due to the propagation channel conditions as the left figure illustrates (neighboring cells present a similar behavior). In consequence, the algorithm proposes a different solution in time, making three femtocells (Femto x1, Femto x3, and Femto x4) fight to get the blue (left icon in Fig. 10) or red mobile phone. The instability of the RSS values from these neighboring cells generates this situation: either the strongest RSS value from neighboring cells is received by the blue mobile phone (from Femto $\mathrm{x} 3 / \mathrm{x} 4$ ) or from the red mobile phone (Femto $\mathrm{x} 1$ ). That means there is not a unique solution for that situation in time; therefore, the number of handovers could be increased (as simulations depict in Fig. 7b).

After that, a real-time indoor-localization system was implemented in order to supply online users' positions to the SON algorithms. This system made use of RSS and orientation information of the terminal to calculate its position. The cumulative density function (CDF) positioning error is $3 \mathrm{~m}$ for the $50 \%$ and $5.9 \mathrm{~m}$ for the $90 \%$.

The HPLM method under this localization inaccuracy proposes similar femtocell transmission power (estimated power variation) to the use case of accurate positions (see Table 2). Once these new transmission powers are tuned in femtocells, users hand over and network is balanced. This follows the expected behavior discussed in Subsection 5.4 as the accuracy of the indoor positioning system is below $5 \mathrm{~m}$ (use case: 4 users/femtocell).

\section{Conclusions}

This work has been proposed for those operators that prioritize users making phone calls over any other service in indoor femtocell scenarios. For that purpose, a mechanism to work under temporary traffic fluctuations in femtocell environment has been implemented in order to avoid or prevent blocking/dropping calls and to enhance the users QoE indoors. How the proposed method works under different femtocell capacities with regard to the number of maximum users has been studied. In parallel, the two methods to obtain the RSS information have been analyzed.

The HPLM method has presented the best source of RSS values to calculate the estimated transmission power. Nevertheless, the accuracy of this algorithm depends on a positioning system where the online user location could be provided with errors (up to several meters). In consequence, the system performance would decrease depending on the location error. The MR method outperforms the previous method when the user location error starts to be over a few meters. The location error threshold to choose the HPLM or the MR method depends on the femtocell capacities.

Future work will be focused on the deployment of these mechanisms in other scenarios like malls with different indoor positioning systems to evaluate the impact of the location accuracy in a real scenario. In addition, the analysis of extra context-aware information in the development of new context-aware load balancing mechanisms indoors will be performed.

\section{Acknowledgements}

This work has been partially funded by the Spanish Ministry of Economy and Competitiveness within the National Plan for Scientific Research,

Technological Development and Innovation 2008-2011 and the European Development Fund (ERDF), within the MONOLOC project (IPT-430000-20111272). This work has been also partially supported by the Junta de Andalucía (Proyecto de Investigación de Excelencia P12-TIC-2905).

\section{About the authors}

Alejandro Aguilar-Garcia graduated in Telecommunication Engineering from the University of Málaga in 2010. He started his career at Sony European Technology Centre in the Speech and Sound Group, participating on an existing video classification system based on audio and image features. He is currently working towards his PhD developing novel SON mechanisms for small cells in mobile networks at the Communications Engineering Department at the University of Málaga.

Sergio Fortes received his M.Sc. degree in Telecommunication Engineering from the University of Málaga in 2008. He began his career being part of the main European space agencies (DLR, CNES, ESA) and Avanti

Communications plc, where he participated in various research and consultant activities on broadband and aeronautical satellite communications. In 2012, he joined the University of Malaga, where he is currently pursuing his Ph.D. focused on Self-Organizing Networks for cellular communications.

Aarón Garrido is a researcher at Alcatel-Lucent in Madrid. He received a degree in Telecommunications from Universidad Carlos III de Madrid (UC3M). Aarón Garrido recently has begun his career at Alcatel-Lucent, where he is involved in radio communications and signal processing activities. He is currently involved in the MONOLOC research project.

Alfonso Fernández-Durán is regional product line manager for $4 \mathrm{G}$ wireless at Alcatel-Lucent Spain. He received an M.Sc. and a Ph.D. in telecommunication engineering from Universidad Politécnica de Madrid, and an International Master's in management from EM Lyon. He began his career at Alcatel's Corporate Research Center, later becoming project manager for LMDS development in Alcatel's Wireless Access Division. He is currently involved in activities related to wireless indoor solutions and wireless small cell solutions. Raquel Barco holds a M.Sc. and a Ph.D. in Telecommunication Engineering from the University of Malaga. She has worked at Telefonica in Madrid (Spain) and the European Space Agency (ESA) in Darmstadt (Germany). In 2000, she joined the University of Malaga, where she is currently Associate Professor. Her research interests include satellite and mobile communications, mainly focusing on Self-Organizing Networks.

\section{Competing interests}

The authors declare that they have no competing interests.

\section{Author details}

'Universidad de Málaga, Andalucía Tech, Campus de Teatinos s/n, 29071 Málaga, Spain. ${ }^{2}$ Alcatel-Lucent, Madrid, Spain. 
Received: 20 March 2016 Accepted: 10 August 2016

Published online: 27 August 2016

\section{References}

1. $\quad 3 G P P$ TS 32.500 V12.1.0, Telecommunication Management; Self-Organizing Networks (SON); Concepts and requirements, 2014

2. H Claussen, LT Ho, LG Samuel, Self-optimization of coverage for femtocell deployments, IEEE Wireless Telecommunications Symposium, 2008, pp. 278285, Pomona, CA, 2008

3. P Munoz, R Barco, J Ruiz-Aviles, I de la Bandera Cascales, A Aguilar-Garcia, Fuzzy rule-based reinforcement learning for load balancing techniques in enterprise LTE femtocells. IEEE Trans. Veh. Technol. 62(5), 1962-1973 (2013)

4. SOCRATES-self-optimisation and self-configuration in wireless networks. [Online]. Available: http://www.fp7-socrates.org/

5. SEMAFOUR. Self-management of unified heterogeneous radio access networks. [Online]. Available: http://www.fp7-semafour.eu/

6. K Lee, H Lee, Y-U Jang, D-H Cho, CoBRA: cooperative beamforming-based resource allocation for self-healing in SON-based indoor mobile communication system. IEEE Trans. Wirel. Commun. 12(11), 5520-5528 (2013)

7. BeFemto. Broadband evolved FEMTO networks. [Online]. Available: http:// www.ict-befemto.eu/

8. S Hämäläinen, H Sanneck, C Sartori, LTE Self-Organising Networks (SON): Network management automation for operational efficiency (Wiley, 2011)

9. JG Andrews, H Claussen, M Dohler, S Rangan, MC Reed, Femtocells: past, present, and future. IEEE J. Sel. Areas Commun. 30(3)، 497-508 (2012)

10. 3GPP TSG-RAN WG2 meeting \#85 R2-140089 "Mobility performance in real network" Qualcomm Incorporation.

11. S Fortes, A Aguilar-Garcia, R Barco, F Barba, JA Fernandez-Luque, A Fernandez-Duran, Management architecture for location-aware selforganizing LTE/LTE—a small cell networks. IEEE Commun. Mag. 53(1), 294302 (2015)

12. 3GPP TR 36.902 V9.3.1, Evolved Universal Terrestrial Radio Access Network (EUTRAN); Self-Configuring and Self-Optimizing Network (SON) Use Cases and Solutions, 2011. Release 9

13. Q Ye, B Rong, Y Chen, M Al-Shalash, C Caramanis, JG Andrews, User association for load balancing in heterogeneous cellular networks. IEEE Trans. Wirel. Commun. 12(6), 2706-2716 (2013)

14. M Sheng, C Yang, Y Zhang, J Li, Zone-based Load Balancing in LTE SelfOptimizing Networks: A Game Theoretic, IEEE Transactions on Vehicular Technology, vol. 63, no. 6, pp. 2916-2925, 2013.

15. P Szilagyi, Z Vincze, C Vulkan, Integrated Mobility Load Balancing and Traffic Steering mechanism in LTE, International Symposium on Personal Indoor and Mobile Radio Communications (PIMRC), 2013 IEEE 24th, London, 8-11 September 2013, pp. 2148-2153

16. A Lobinger, S Stefanski, T Jansen, I Balan, Load balancing in downlink LTE self-optimizing networks, Vehicular Technology Conference (VTC 2010Spring), 2010 IEEE 71st, Taipei, 16-19 May 2010, pp. 1-5

17. JM Ruiz-Avilés, M Toril, S Luna-Ramírez, A femtocell location strategy for improving adaptive traffic sharing in heterogeneous LTE networks. EURASIP J. Wirel. Commun. Netw. 2015, 38 (2015)

18. Z Becvar, P Mach, Adaptive hysteresis margin for handover in femtocell networks, in Wireless and Mobile Communications (ICWMC), 2010

19. JM Ruiz-Avilés, S Luna-Ramírez, M Toril, F Ruiz, Traffic steering by self-tuning controllers in enterprise LTE femtocells. EURASIP J. Wirel. Commun. Netw. 2012, 337 (2012)

20. A Aguilar-Garcia, R Barco, S Fortes, P Muñoz, Load balancing mechanisms for indoor temporary overloaded heterogeneous femtocell networks. EURASIP J. Wirel. Commun. Netw. 29, 2015 (2015)

21. J Steuer, K Jobmann, The use of mobile positioning supported traffic density measurements to assist load balancing methods based on adaptive cell sizing, In Proceedings of IEEE International Symposium on Personal, Indoor and Mobile Radio Communications (PIMRC), 15-18 September 2002, pp. 339343

22. NA Amirrudin, SHS Ariffin, NNNA Malik, NE Ghazali, User's mobility historybased mobility prediction in LTE femtocells network, RF and Microwave Conference (RFM), 2013 IEEE International, Penang, 9-11 December 2013, pp. 105-110

23. A Freedman, D Dilmon, A Assayag, E Deutscher, Prediction based RSS fingerprinting for positioning and optimization in cellular networks, Electrical \& Electronics Engineers in Israel (IEEEI), 2012 IEEE 27th Convention of, Eilat, 14-17 November 2012, pp. 1-4
24. F Guidolin, I Pappalardo, A Zanella, M Zorzi, A Markov-based framework for handover optimization in HetNets, Ad Hoc Networking Workshop (MEDHOC-NET), 2014 13th Annual Mediterranean, Piran, 2-4 June 2014, pp. 134139

25. H Li, S Habibi, G Ascheid, Handover prediction for long-term window scheduling based on SINR maps, Personal Indoor and Mobile Radio Communications (PIMRC), 2013 IEEE 24th International Symposium on, London, 8-11 September 2013, pp. 917-921

26. S Donghoon, C Sunghee, Dynamic power control for balanced data traffic with coverage in femtocell networks, Wireless Communications and Mobile Computing Conference (IWCMC), 2012 8th International, Limassol, 27-31 August 2012, pp. 648-653

27. 3GPP specification; Evolved Universal Terrestrial Radio Access (E-UTRA); radio resource control (RRC); protocol specification (3GPP TS 36.331 version 12.3.0 Release 12)

28. 3GPP specification; Evolved Universal Terrestrial Radio Access (E-UTRA); requirements for support of radio resource management (3GPP TS 36.133 version 12.3.0 Release 12)

29. K Subbu, C Zhang, J Luo, A Vasilakos, Analysis and status quo of smartphone-based indoor localization systems. IEEE Wirel. Commun. 21(4), 106-112 (2014)

30. JM Ruiz-Avilés, S Luna-Ramírez, M Toril, F Ruiz, I de la Bandera, P Muñoz, R Barco, P Lázaro, V Buenestado, Design of a computationally efficient dynamic system-level simulator for enterprise LTE femtocell scenarios. J. Electr. Comput. Eng. 2012, 14 (2012)

31. D1.1.2. WINNER II channel models. Part II. Radio channel measurement and analysis results. v1.0 Tech. rep. WINNER II IST project (2007)

32. T Sorensen, P Mogensen, F Frederiksen, Extension of the ITU channel models for wideband (OFDM) systems, Vehicular Technology Conference, 2005. VTC-2005-Fall. 2005 IEEE 62nd, 25-28 September 2005, pp. 392-396

33. C Bettstetter, C Wagner, The spatial node distribution of the random waypoint mobility model, In Proceedings of German Workshop on Mobile Ad Hoc networks (WMAN), March 2002

34. C Feng, WSA Au, S Valaee, Z Tan, Received-signal-strength-based indoor positioning using compressive sensing. IEEE Trans. Mob. Comput. 11(12), 1983-1993 (2012)

35. A Rai, KK Chintalapudi, VN Padmanabhan, R Sen, Zee: Zero-Effort Crowdsourcing for Indoor Localization, Proceedings of the 18th annual international conference on Mobile computing and networking, pp. 293-304, 2012

36. Alcatel Lucent 9361 Home Cell v2' Datasheet. [Online]. Available: http:// www3.alcatel-lucent.com/wps/DocumentStreamerServlet?LMSG_ CABINET=Docs_and_Resource_Ctr\&LMSG_CONTENT_FILE=Data_Sheets/ Home_Cell_V2_2100_MHz_EN_DataSheet.pdf

\section{Submit your manuscript to a SpringerOpen ${ }^{\mathcal{O}}$ journal and benefit from:}

- Convenient online submission

- Rigorous peer review

- Immediate publication on acceptance

- Open access: articles freely available online

- High visibility within the field

- Retaining the copyright to your article

Submit your next manuscript at springeropen.com 